\title{
Evidence of response to pembrolizumab in a patient with Lynch syndrome-related metastatic colon cancer
}

This article was published in the following Dove Press journal: OncoTargets and Therapy

\section{Pamela Salman' \\ Sergio Panay' \\ René Fernández ${ }^{2}$ \\ Mauricio Mahave' \\ Cristian Soza-Ried'}

'Department of Medical Oncology, Instituto Oncológico Fundación Arturo López Pérez, Santiago, Chile; ${ }^{2}$ Department of Nuclear Medicine, Instituto Oncológico Fundación Arturo López Pérez, Santiago, Chile

Video abstract

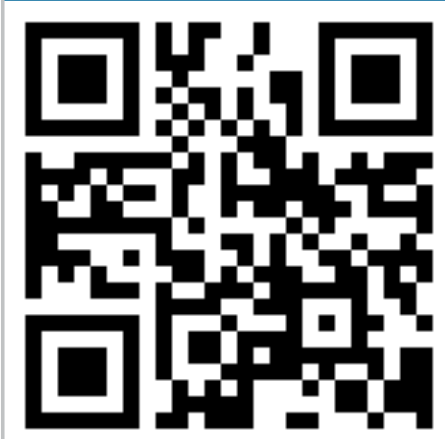

Point your SmartPhone at the code above. If you have a QR code reader the video abstract will appear. Or use:

http://youtu.be/hWot3v719YY

Correspondence: Pamela Salman Oncología Médica, Instituto Oncológico Fundación Arturo López Pérez, Rancagua 878, Providencia, Región Metropolitana

750092I, Santiago, Chile

$\mathrm{Tel}+56224205$ I 5 I

Fax +56 2242I 8596

Email salmanp@falp.org

\begin{abstract}
Patients with Lynch Syndrome (LS) are at high risk of developing colorectal cancer at an early age. Germline mutations in DNA mismatch repair genes and microsatellite instability are clear signatures of this autosomal dominant disorder. Here, we report the clinical history of a 38-year-old patient with LS-related metastatic colon cancer treated in Chile with immunotherapy (pembrolizumab). The patient exhibited a pathogenic deletion in Epithelial cell Adhesion Molecule (EPCAM) and mutS homolog 2 (MSH2) genes, and after diagnosis received 12 cycles of FOLFOX. The tumor mass, however, continued to grow, and a new metastatic mucinous adenocarcinoma of $13 \mathrm{~mm}$ appeared at the level of the 11th right dorsal vertebra. To treat these lesions, the patient received immunotherapy scheme with pembrolizumab (200 mg every 21 days). After only four cycles, the patient's symptoms improved and the lesions showed less metabolic activity. After 12 cycles with pembrolizumab, the patient started palliative radiation and systemic second-line treatment with FOLFIRI and Avastin. The immunotherapy scheme with pembrolizumab was capable of delaying the second-line treatment for at least 8 months, becoming a useful therapeutic option for this patient. Thus, our study highlights the importance of implementing immunotherapy treatment programs for LS-colorectal cancer patients in South American countries.
\end{abstract}

Keywords: immunotherapy, DNA mismatch repair proteins, EPCAM, MSH2

\section{Introduction}

Mutations in genes that encode for DNA mismatch repair (MMR) proteins results in a systematic accumulation of DNA replication errors. In time, these mutations may evolve leading to tumor development. Short repetitive nucleotide sequences, known as microsatellite, are susceptible targets for these replication errors, a condition called microsatellite instability (MSI). ${ }^{1,2}$ Loss of MMR protein functions results in hereditary nonpolyposis colorectal cancer (HNPCC). ${ }^{3}$ However, when HNPCC develops as a consequence of germline mutations in at least one of the genes that encode proteins implicated in the MMR system, the pathology is classified as Lynch syndrome (LS). Thus, mutations in mutS homolog 2 (MSH2), mutS homolog 3 (MSH3), mutS homolog 6 (MSH6), mutL homolog 1 (MLH1), PMS1 homolog 1, mismatch repair system component ( $P M S 1$, also known as $M L H 2$ ), and PMS1 homolog 2, mismatch repair system component (PMS2, also known as MLH4) are usually involved in LS. ${ }^{3-10} \mathrm{LS}$ is considered the most common condition among the inherited colorectal cancers and is responsible for $2 \%-4 \%$ of all colorectal carcinomas (CRC)., ${ }^{3,4,7,11-13}$ This syndrome corresponds to an autosomal dominant disorder. Therefore, first-degree relatives have up to $50 \%$ risk of inheriting the mutation, and depending on the mutation penetrance, 
several members of their family may also be clinically affected. Thus, patient's clinical information is complemented with family history according to the Amsterdam criteria and the Bethesda guidelines. ${ }^{14} \mathrm{LS}$ patients are at high risk of developing CRC (up to $75 \%$ ), endometrial cancer (32\%-45\%), stomach cancer (13\%-19\%), and ovarian cancer $(9 \%-14 \%)^{3}{ }^{3,5,14,15}$ But, the risk of developing cancer in other tissues such as small intestine, pancreas, biliary and urinary tract, brain (glioblastomas), skin (keratoacanthomas), and sebaceous tissues are also high., ${ }^{3,5,7,9,12,13,16}$ Furthermore, cancer develops at a relatively young age in LS patients, and in the case of CRC, the neoplasm progression is rather fast. ${ }^{17}$

In LS deletions, frameshifts, nonsense, missense, and splice mutations are frequent in $M S H 2$ and $M L H 1$ genes, whereas mutations in MSH6 and PMS2 represent $<10 \%$. . $^{3,9}$ MSH2 loss of function impairs the recognition of base-base mismatch and insertion-deletion loops, which depends on the heterodimer formation between MSH2 and MSH6 or MSH2 and MSH3. Meanwhile, the repair of base-base mismatches and insertion-deletion loops depends mainly on MLH1, PSM1, and PMS2 proteins. ${ }^{18}$

Another gene implicated in LS is Epithelial cell Adhesion Molecule (EPCAM; OMIM\#185535, previously known as TACSTD1), whose deletion produces allele-specific methylation of MSH2 (OMIM\#609309) gene promoter, precluding MSH2 expression. ${ }^{3-5,12}$ Despite the broad spectrum and particular frequency of pathogenic MMR variants in the Latin America population, only a few cases have been described. ${ }^{14}$

According to FIRE 3 and TRIBE trials, patients with metastatic CRC (mCRC) or nonresectable CRC have an overall survival of 28.7-37.1 months. ${ }^{19,20}$ Those patients can follow a first-line treatment, which consists of chemotherapy (either infused fluoropyrimidine plus oxaliplatin or irinotecan) in combination with a biological agent. Both chemotherapy regimens have similar efficiency. Therefore, the selection of either of the chemotherapy regimens depends on patient's differential toxicity profile. Likewise, the choice of the biological agent will depend on patient's molecular profile and the absence of contraindications. ${ }^{21}$ The second-line treatment depends on patient's organ function and cytotoxicity tolerance and the first-line therapy choice. A third-line treatment considers cetuximab or panitumumab for $R A S$ and $B R A F$ wild-type patients and EGFR antibody for patients who have not used that antibody previously. Regorafenib (multikinase inhibitor) or Trifluridine/tipiracil is recommended for $R A S$ wild-type patients treated with EGFR antibodies and in patients pretreated with fluoropyrimidines, oxaliplatin, irinotecan, and bevacizumab. ${ }^{22}$
Patients with LS show flat tumors located in the proximity of splenic flexure with poor differentiation and lymphocytic infiltration. ${ }^{19}$ Likewise, these tumors present a microenvironment populated by myeloid cells expressing the programmed death ligand 1 (PD-L1), which impair the function of the infiltrated $\mathrm{CD} 8^{+} \mathrm{T}$ and T-helper 1 cells. The immunological blockade can be bypassed, however, by using monoclonal antibodies against PD-1. The usage of PD-1 antibodies has shown promising results in other cancers such as melanoma and non-small cell lung cancer. ${ }^{23-27}$ Surprisingly, in the case of CRC patients, anti-PD-1 immunotherapy shows different outcomes. Thus, patients whose tumors exhibit mismatch repair-deficiencies are more likely to respond than those whose tumors lack mutations in mismatch repair genes. ${ }^{28,29}$ This finding opened a new therapeutic door to treat LS patients based on the molecular signature rather than the type of tumor. ${ }^{30}$ In this study, we report a male with LS-related colon cancer, who exhibits pathogenic deletion involving both EPCAM and MSH2. The patient, treated in Chile, received an immunotherapy scheme with pembrolizumab (Keytruda) that delayed the second-line treatment with FOLFIRI and Avastin for at least 8 months. This emphasizes the importance of including LS-colorectal cancer patients in immunotherapy treatment programs in Latin America.

\section{Case report}

The patient gave written informed consent to publish this case report and associated images. The clinical history of the following report corresponds to a patient aged 38 years, at the time of diagnosis. He had a car accident in 2004, which resulted in secondary transient erythroblastopenia with recovered hemiparesis. In January 2015, he underwent surgery to remove his appendix due to acute appendicitis. A biopsy was performed detecting an adenocarcinoma including serous tissue. An analysis of the family history revealed that his father had been diagnosed with colon cancer and skin carcinoma. At the end of March, the patient was referred to Instituto Oncologico Fundación Arturo López Pérez in Chile for further analysis. A blood test revealed an elevated level of the carcinoembryonic antigen (CEA of 237), and an exploratory laparotomy found tumors in the ascending colon and retroperitoneal space. An R0 surgery was planned, and to that end, positron emission tomography-computed tomography (PET/CT) scan was used to define the surgery area to the right side of the colon. The R0 surgery removed the parietal peritoneum including tumor masses from the retroperitoneum.

The analysis of the biopsy showed a poorly differentiated mucinous adenocarcinoma of $10 \mathrm{~cm}$ in the cecum and a second poorly differentiated tubular adenocarcinoma in the appendix 
implantation base with an increased lymphatic vessel permeability and infiltration until adventitia. A third $2 \mathrm{~cm}$ tumor mass was found in the ileocecal valve corresponding to a poorly differentiated mucinous adenocarcinoma with 21/31 positive lymph nodes. Likewise, the retroperitoneal mass was with a poorly differentiated mucinous adenocarcinoma.

An immunohistochemistry (IHC) analysis performed in April showed lack of MSH2 and EPCAM expression and the wild-type expression of KRAS-NRAS. To analyze the presence of MSI, a fluorescent PCR-based assay was performed (Promega MSI Analysis System). To this end, DNA samples were obtained from patient's formalin-fixed paraffin-embedded tissue. Unfortunately, quality of the extracted DNA was insufficient for the assay. Although MMR proteins screening by IHC and microsatellite genotyping have similar effectiveness, ${ }^{7,31}$ MSI and IHC analysis are considered complementary to one another. ${ }^{32}$ To characterize the lack of MMR protein expression, further genomic sequencing and deletion/duplication studies were conducted. The patient's DNA sample was enriched for targeted regions using hybridization-based protocol, and sequencing was performed by using illumine technology (targeted regions were sequenced with $\geq 50 \times$ depth).

The analysis of genomic and deletion/duplication studies revealed a pathogenic heterozygous deletion involving both the EPCAM and MSH2 genes, which are located on the short (p) arm of chromosome 2 at position 21. All of the EPCAM's nine exons were deleted (EX1-EX9del), whereas neighboring gene $\mathrm{MSH} 2 \mathrm{had}$ a deletion comprising exons 1-6 (EX1EX6del). Since deletion of both genes is known to cause
LS, the mutations were considered pathogenic. Other related genes, such as MLH1, MSH6, and PMS2, were screened; however, the results were negative. Likewise, no mutation was found in KRAS (KRAS/BRAF Mutation Analysis Panel Kit for Real-Time PCR, exons 2, 3, and 4 of KRAS and V600E of BRAF; EntroGen; Woodland Hills, CA, USA) or NRAS (NRAS Mutation Detection Kit, EntroGen). The patient received adjuvant treatment with 12 cycles of FOLFOX (leucovorin, fluorouracil [5FU], and oxaliplatin) until November 29, 2015. After finishing the cycles of FOLFOX, the patient exhibited a reduction in CEA levels (from 237 in March 2015 to 27.9 in March 2016, not shown). However, a more detail analysis using PET/CT ${ }^{18} \mathrm{~F}$-FDG scan revealed tumor lesions at two different locations: the intercostal nodule at the level of the 12th right rib (Figure 1A-C) and a retrocrural adenopathy at the level of the 11th right dorsal vertebra (Figure 2A-C). The first PET/CT ${ }^{18} \mathrm{~F}-\mathrm{FDG}$ scan after the 12 cycles of FOLFOX, conducted in April 2016, showed that the tumor mass at the intercostal nodule had reached $24 \mathrm{~mm}$ (Figure 1A) and revealed a retrocrural adenopathy of $13 \mathrm{~mm}$ at the level of the 11th right dorsal vertebra (Figure 2A).

The biopsy confirmed that this new lesion corresponded to a metastatic mucinous adenocarcinoma. To treat these painful intercostal lesions, a new treatment scheme to block PD-1 with pembrolizumab (200 mg every 21 days) was implemented on June 23, 2016. After four cycles, the CEA levels were normalized (not shown), and the patient's symptoms improved. We observed a slight size increase of the retrocrural adenopathy (at the 11th right dorsal vertebra) from 13 to
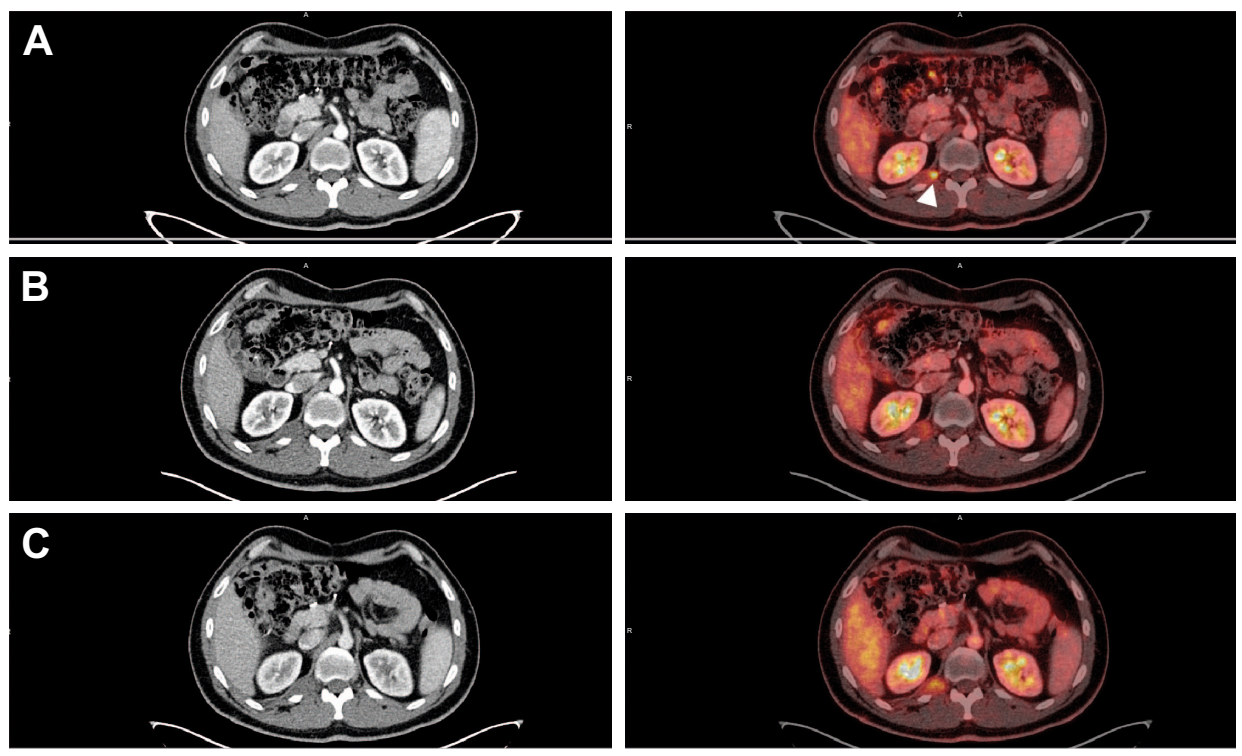

Figure I PET/CT ${ }^{18} \mathrm{~F}-\mathrm{FDG}$ scan of tumor mass at the intercostal nodule at the level of the 12 th right rib.

Notes: CT and the corresponding PET image obtained in April, 20I6; the arrow indicates metabolic activity of the lesion (SUV[max] value of 5.3) (A). CT and the corresponding PET image obtained in August (B) and in December (C).

Abbreviations: PET/CT, positron emission tomography-computed tomography; SUV, standardized uptake value. 

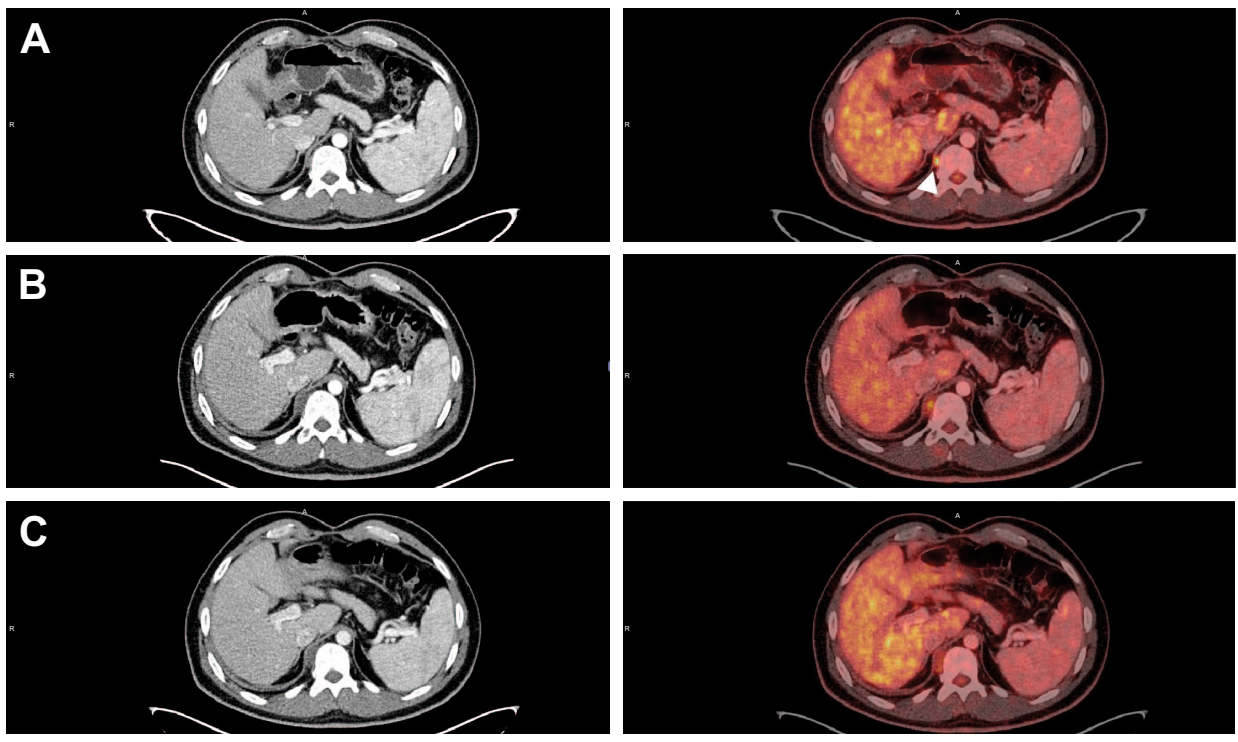

Figure 2 PET/CT ${ }^{18}$ F-FDG scan of the retrocrural adenopathy at the level of the II th right dorsal vertebra.

Notes: CT and the corresponding PET image obtained in April; the arrow indicates metabolic activity of the lesion (SUV[max] value of 5.2) (A). CT and the corresponding PET image obtained in August (B) and in December (C).

Abbreviations: PET/CT, positron emission tomography-computed tomography; SUV, standardized uptake value.

$16 \mathrm{~mm}$ and the intercostal nodule (at the level of the 12th right rib) from 24 to $26 \mathrm{~mm}$. However, both lesions showed less metabolic activity according to the PET/CT Standardized Uptake Value (SUV) (SUV[max] values of 4 and 3.2 instead of the previous 5.2 and 5.3, respectively; Figures $1 \mathrm{~A}$ and $\mathrm{B}$ and $2 \mathrm{~A}$ and $\mathrm{B})$. These promising results led to six more cycles. In December, a new medical evaluation showed that the hypodense retrocrural nodule at the 11th right dorsal vertebra increased in size from 16 to $22 \mathrm{~mm}$. Despite this, the metabolic activity from the previous $\mathrm{SUV}(\max )$ value decreased from 4 to 3.5 (Figure 2B and C). Similarly, the intercostal nodule at the level of the 12th right rib experimented a slight increase from 26 to $28 \mathrm{~mm}$; however, its metabolic activity rose from an SUV(max) value of 3.2 to 4.1 (Figure $1 \mathrm{~B}$ and $\mathrm{C}$ ). The patient followed two more cycles of pembrolizumab until February 2017, thus completing 12 cycles. In April 2017, he underwent palliative radiation and systemic therapy with FOLFIRI (Leucovorin Calcium, Fluorouracil and Irinotecan Hydrochloride) and Avastin, which continues until today.

\section{Discussion}

In this report, we expose the case of a patient with LS, harboring a deletion of all exons of EPCAM gene, which compromise the exons 1-6 from the neighbor $M S H 2$. Deletion of EPCAM is present only in a subgroup of LS patients; however, this inactivation usually drives epigenetic silencing of $\mathrm{MSH} 2$ rather than deletion of this gene. ${ }^{33}$ The EPCAM-MSH2 inactivation favors the appearance of other malignancies such as endometrial cancer. ${ }^{34}$ Rossi et al showed that in Latin America, pathogenic variants of $M S H 2$ are frequent, especially in Argentina. ${ }^{14}$ Usually, these pathogenic variants are due to single nucleotide changes that produce frameshifts, missense and nonsense mutations. The most affected regions in $\mathrm{MSH} 2$ are exons $3(17 \%)$ and 7 (15\%), whereas our patient shows a large deletion compromising exons 1-6. Large deletions in EPCAM - affecting the 3 ' region - are frequent, although, its prevalence in the Latin American population is relatively low. ${ }^{14}$ Interestingly, our patient presented a large deletion compromising the function of both EPCAM and $\mathrm{MSH} 2$ genes. This mutation abolished the expression and function of MSH2 protein leading to the development of LS.

Recently, the therapeutic potential of antibodies to target relevant immunologic checkpoints in mismatch repairdeficient colon cancer patients has been scrutinized. ${ }^{26-28}$ Le et $\mathrm{al}^{29}$ evaluated the clinical response to pembrolizumab in 41 patients with progressive metastatic carcinoma. The study compared between colorectal cancer patients with mismatch repair-deficient tumors (cohort A), patients with mismatch repair-proficient colorectal adenocarcinomas or microsatellite-stable cancers (cohort B), and patients with mismatch repair-deficient non-colon cancers (cohort $\mathrm{C}$ ). Patients with mismatch repair-deficient cancers were more responsive to PD-1 blockade than those without mismatch repair-deficient cancers. In fact, $40 \%$ of the patients from cohort A had an immune-related objective response rate $(95 \% \mathrm{CI}, 12-74)$ according to the RECIST criteria. 
Seventy-one percent of cohort $\mathrm{C}$ patients had an immunerelated objective response rate (95\% CI, 29-96) and responded faster than patients from cohort A (12 weeks vs 28 weeks). On the contrary, patients from cohort B failed to show an immune-related objective response (95\% CI, 0-19) as defined by RECIST. Moreover, the study showed that pembrolizumab treatment decreased the levels of CEA in patients with mismatch repair-deficient colorectal cancers, which was not the case for patients without mismatch repair-deficiency. ${ }^{29}$ Our results support this observation as after four cycles with pembrolizumab the CEA levels in our patient were normalized.

A more recent study showed that a female patient with sporadic mismatch repair-deficient mCRC and bulky abdominal disease treated with pembrolizumab had a remarkable response. ${ }^{28}$ Tumors with MMR deficiency have an elevated mutational load and, as a consequence, significant numbers of tumor-infiltrating lymphocytes in the tumor microenvironment. ${ }^{26}$ Thus, PD-1 expression on lymphocytes and PD-L1 on tumor cells and the numbers of mutation-associated neoantigens are high in comparison with microsatellite-stable tumors. ${ }^{29,35}$

In this report, we describe the case of an LS-colorectal cancer patient, with mutations in EPCAM and MSH2. The patient was treated with pembrolizumab according to the molecular status of his cancer. This immunotherapy scheme delayed the second-line treatment with FOLFIRI and Avastin for at least 8 months, demonstrating to be an important therapeutic option. Although, the responsiveness of LS-colorectal cancer patients to pembrolizumab has been well documented, in South American countries it is not a standard procedure. Usually after diagnosis, LS patients follow a standard $\mathrm{mCRC}$ chemotherapy scheme as immunotherapy is expensive and not covered by public health insurances. Our results highlight the importance for South American countries to include LS-colorectal cancer patients in immunotherapy treatment programs. Especially, considering that a therapeutic scheme with pembrolizumab is less toxic than chemotherapy and has the potential to provide a durable response. The anti-PD-1 response of mismatch repairdeficient cancers is not restricted to LS colorectal cancers. ${ }^{27}$ Therefore, the identification of MMR-deficient cancers will improve diagnostic and treatment strategies.

\section{Acknowledgments}

We owe thanks to the patient and his family and to the Instituto Oncológico Fundación Arturo López Pérez for supporting the publication of this work. Present address for Cristian Soza-ried is at Escuela de Bioquímica, Facultad de Ciencia, Universidad
San Sebastián and Instituto Oncológico Fundación Arturo López Pérez, Santiago, Región Metropolitana, Chile.

\section{Author contributions}

PS designed the study. CS-R and PS planned and wrote the paper. PS, SP, and MM treated the patient and RF performed the PET/CT ${ }^{18} \mathrm{~F}$-FDG scans. All authors contributed toward data analysis, drafting and revising the paper and agree to be accountable for all aspects of the work.

\section{Disclosure}

The authors report no conflicts of interest in this work.

\section{References}

1. Ionov Y, Peinado MA, Malkhosyan S, Shibata D, Perucho M. Ubiquitous somatic mutations in simple repeated sequences reveal a new mechanism for colonic carcinogenesis. Nature. 1993;363(6429): 558-561.

2. Thibodeau SN, Bren G, Schaid D. Microsatellite instability in cancer of the proximal colon. Science. 1993;260(5109):816-819.

3. Carethers JM, Stoffel EM. Lynch syndrome and Lynch syndrome mimics: the growing complex landscape of hereditary colon cancer. World J Gastroenterol. 2015;21(31):9253-9261.

4. Carethers JM. Differentiating Lynch-like from Lynch syndrome. Gastroenterology. 2014;146(3):602-604.

5. Stoffel EM, Kastrinos F. Familial colorectal cancer, beyond Lynch syndrome. Clin Gastroenterol Hepatol. 2014;12(7):1059-1068.

6. Grady WM, Carethers JM. Genomic and epigenetic instability in colorectal cancer pathogenesis. Gastroenterology. 2008;135(4):1079-1099.

7. Hampel H, Frankel WL, Martin E, et al. Screening for the Lynch syndrome (hereditary nonpolyposis colorectal cancer). N Engl J Med. 2005;352(18):1851-1860.

8. Wells K, Wise PE. Hereditary colorectal cancer syndromes. Surg Clin North Am. 2017;97(3):605-625.

9. Kheirelseid EA, Miller N, Chang KH, et al. Mismatch repair protein expression in colorectal cancer. J Gastrointest Oncol. 2013;4(4): 397-408.

10. Dudley JC, Lin MT, Le DT, Eshleman JR. Microsatellite instability as a biomarker for PD-1 blockade. Clin Cancer Res. 2016;22(4):813-820.

11. Jenkins MA, Baglietto L, Dowty JG, et al. Cancer risks for mismatch repair gene mutation carriers: a population-based early onset case-family study. Clin Gastroenterol Hepatol. 2006;4(4):489-498.

12. Boland CR, Koi M, Chang DK, Carethers JM. The biochemical basis of microsatellite instability and abnormal immunohistochemistry and clinical behavior in Lynch syndrome: from bench to bedside. Fam Cancer. 2008;7(1):41-52.

13. Hampel H, Frankel W, Panescu J, et al. Screening for Lynch syndrome (hereditary nonpolyposis colorectal cancer) among endometrial cancer patients. Cancer Res. 2006;66(15):7810-7817.

14. Rossi BM, Palmero EI, López-Kostner F, et al. A survey of the clinicopathological and molecular characteristics of patients with suspected Lynch syndrome in Latin America. BMC Cancer. 2017;17(1):623.

15. Stoffel EM. Heritable gastrointestinal cancer syndromes. Gastroenterol Clin North Am. 2016;45(3):509-527.

16. Kobayashi H, Ohno S, Sasaki Y, Matsuura M. Hereditary breast and ovarian cancer susceptibility genes (review). Oncol Rep. 2013;30(3): 1019-1029.

17. Järvinen HJ, Aarnio M, Mustonen H, et al. Controlled 15-year trial on screening for colorectal cancer in families with hereditary nonpolyposis colorectal cancer. Gastroenterology. 2000;118(5):829-834.

18. Jiricny J. Replication errors: cha(lle)nging the genome. EMBO J. 1998;17(22):6427-6436. 
19. Heinemann V, von Weikersthal LF, Decker T, et al. FOLFIRI plus cetuximab versus FOLFIRI plus bevacizumab as first-line treatment for patients with metastatic colorectal cancer (FIRE-3): a randomised, open-label, phase 3 trial. Lancet Oncol. 2014;15(10):1065-1075.

20. Cremolini C, Loupakis F, Falcone A. FOLFOXIRI and bevacizumab for metastatic colorectal cancer. N Engl J Med. 2015;372(3):291-292.

21. Mahipal A, Grothey A. Role of biologics in first-line treatment of colorectal cancer. J Oncol Pract. 2016;12(12):1219-1228.

22. van Cutsem E, Cervantes A, Adam R, et al. ESMO consensus guidelines for the management of patients with metastatic colorectal cancer. Ann Oncol. 2016;27(8):1386-1422.

23. Hodi FS, O'Day SJ, Mcdermott DF, et al. Improved survival with ipilimumab in patients with metastatic melanoma. $N$ Engl J Med. 2010;363(8):711-723.

24. Brahmer JR. PD-1-targeted immunotherapy: recent clinical findings. Clin Adv Hematol Oncol. 2012;10(10):674-675.

25. Janakiram M, Pareek V, Cheng H, Narasimhulu DM, Zang X. Immune checkpoint blockade in human cancer therapy: lung cancer and hematologic malignancies. Immunotherapy. 2016;8(7):809-819.

26. Lee V, Murphy A, Le DT, Diaz LA. Mismatch repair deficiency and response to immune checkpoint blockade. Oncologist. 2016;21(10): 1200-1211.

27. Le DT, Durham JN, Smith KN, et al. Mismatch repair deficiency predicts response of solid tumors to PD-1 blockade. Science. 2017; 357(6349):409-413.
28. Kieler M, Scheithauer W, Zielinski CC, Chott A, Al-Mukhtar A, Prager GW. Case report: impressive response to pembrolizumab in a patient with mismatch-repair deficient metastasized colorectal cancer and bulky disease. ESMO Open. 2016;1(6):e000084.

29. Le DT, Uram JN, Wang H, et al. PD-1 blockade in tumors with mismatchrepair deficiency. $N$ Engl J Med. 2015;372(26):2509-2520.

30. Lee V, Le DT. Efficacy of PD-1 blockade in tumors with MMR deficiency. Immunotherapy. 2016;8(1):1-3.

31. Barnetson RA, Tenesa A, Farrington SM, et al. Identification and survival of carriers of mutations in DNA mismatch-repair genes in colon cancer. N Engl J Med. 2006;354(26):2751-2763.

32. Umar A, Boland CR, Terdiman JP, et al. Revised Bethesda Guidelines for hereditary nonpolyposis colorectal cancer (Lynch syndrome) and microsatellite instability. J Natl Cancer Inst. 2004;96(4):261-268.

33. Ligtenberg MJ, Kuiper RP, Chan TL, et al. Heritable somatic methylation and inactivation of MSH2 in families with Lynch syndrome due to deletion of the 3' exons of TACSTD1. Nat Genet. 2009;41(1): $112-117$.

34. Kempers MJ, Kuiper RP, Ockeloen CW, et al. Risk of colorectal and endometrial cancers in EPCAM deletion-positive Lynch syndrome: a cohort study. Lancet Oncol. 2011;12(1):49-55.

35. Gatalica Z, Snyder C, Maney T, et al. Programmed cell death 1 (PD-1) and its ligand (PD-L1) in common cancers and their correlation with molecular cancer type. Cancer Epidemiol Biomarkers Prev. 2014;23(12):2965-2970.
OncoTargets and Therapy

\section{Publish your work in this journal}

OncoTargets and Therapy is an international, peer-reviewed, open access journal focusing on the pathological basis of all cancers, potential targets for therapy and treatment protocols employed to improve the management of cancer patients. The journal also focuses on the impact of management programs and new therapeutic agents and protocols on

\section{Dovepress}

patient perspectives such as quality of life, adherence and satisfaction. The manuscript management system is completely online and includes a very quick and fair peer-review system, which is all easy to use. Visit http://www.dovepress.com/testimonials.php to read real quotes from published authors. 